\title{
The Mechanisms of Generation and Propagation of Synchronized Bursting in Developing Networks of Cortical Neurons
}

\author{
Eisaku Maeda, ${ }^{1}$ Hugh P. C. Robinson, ${ }^{2}$ and Akio Kawana' \\ ${ }^{1}$ NTT Basic Research Laboratories, Atsugi-shi, 243-01, Japan, and ${ }^{2}$ Physiological Laboratory, University of \\ Cambridge, Cambridge CB2 3EG, United Kingdom
}

\begin{abstract}
The characteristics and mechanisms of synchronized firing in developing networks of cultured cortical neurons were studied using multisite recording through planar electrode arrays (PEAs). With maturation of the network (from 3 to $40 \mathrm{~d}$ after plating), the frequency and propagation velocity of bursts increased markedly (approximately from 0.01 to $0.5 \mathrm{~Hz}$ and from 5 to $100 \mathrm{~mm} / \mathrm{sec}$, respectively), and the sensitivity to extracellular magnesium concentration (0-10 $\mathrm{mm}$ ) decreased. The source of spontaneous bursts, estimated from the relative delay of onset of activity between electrodes, varied randomly with each burst. Physical separation of synchronously bursting networks into several parts using an ultraviolet laser, divided synchronous bursting into different frequencies and phases in each part. Focal stimulation through the PEA was effective at multiple sites in eliciting bursts, which propagated over the network from the site of stimulation. Stimulated bursts exhibited both an absolute refractory period and a relative refractory period, in which partially propagating bursts could be elicited. Periodic electrical stimulation (at 1 to $30 \mathrm{sec}$ intervals) produced slower propagation velocities and smaller numbers of spikes per burst at shorter stimulation intervals. These results suggest that the generation and propagation of spontaneous synchronous bursts in cultured cortical neurons is governed by the level of spontaneous presynaptic firing, by the degree of connectivity of the network, and by a distributed balance between excitation and recovery processes.
\end{abstract}

[Key words: cultured network, cortical neuron, synchronization, multisite recording, multisite stimulation, planar electrode array, periodic burst, refractoriness, synaptic transmitter]

Spontaneous synchronized neuronal activity has been implicated in the development of the visual system (Shatz, 1990; Meister et al., 1991; Yuste et al., 1992; Wong et al., 1993), in the integration of sensory information (Engel et al., 1992), and in epileptogenesis (Gutnick et al., 1982). In cultured networks of hippocampal and cortical neurons, periodic synchronized calcium

\footnotetext{
Received Mar. 27, 1995; revised June 14, 1995; accepted June 19, 1995.

We are grateful to Drs. M. Meister and V. Torre for valuable comments on the manuscript, Dr. Y. Kuroda for helpful discussions, and S. Sakata for fabrication of electrode arrays. This work was supported by a joint Human Frontier Science Program Research Grant (RG-89/94), a Wellcome Trust Vision Research Fellowship (H.P.C.R.), and a Royal Society Research Grant (H.P.C.R.)

Correspondence should be addressed to Eisaku Maeda, 4S-409S, NTT Basic Research Laboratories, 3-1 Morinosato Wakamiya, Atsugi-shi, 243-01, Japan. Copyright (C) 1995 Society for Neuroscience $0270-6474 / 95 / 156834-12 \$ 05.00 / 0$
}

transients at frequencies between 0.1 and $1 \mathrm{~Hz}$ are observed under conditions of low extracellular magnesium concentration $\left(\left[\mathrm{Mg}^{2+}\right]_{o}\right.$ ) (Ogura et al., 1987; Kuroda et al., 1992; Murphy et al., 1992). These synchronized $\mathrm{Ca}^{2+}$ transients are due to propagation of bursts of action potentials, which are generated periodically and are accompanied by slower $\mathrm{Ca}^{2+}$ transients (Robinson et al., 1992, 1993). Similar bursting activity has been observed in layer 5 of intact neocortical slices in nominally zero $\left[\mathrm{Mg}^{2+}\right]_{o}$ (Silva et al., 1991), in dissociated cortical cultures (Huettner and Baughman, 1986; Ilabets et al., 1987) and organotypic cortical slice cultures in normal, serum-containing medium (Charlety et al., 1994), and in rat hippocampus and cat neocortex in vivo (Buzsáki et al., 1992; Steriade et al., 1993). Remarkable features of the spontaneous bursts and $\mathrm{Ca}^{2+}$ transients in low magnesium include their stable mean frequency and the long quiescent states between bursts. The frequency and propagation velocity of the bursts depend sharply on $\left[\mathrm{Mg}^{2+}\right]_{0}$ and on the degree of network maturation (Kamioka et al., 1993; Maeda et al., 1993b). In addition, bursts elicited by electrical stimulation can produce persistent modification of the frequency and extent of spontaneous bursting (Kuroda et al., 1993; Maeda et al., 1993a). The mechanisms that determine these properties are not yet clear.

This periodic bursting represents a relatively simple and stable mode of activity in networks of cortical neurons, and offers an opportunity to study basic mechanisms of network firing, such as how synchronization is achieved, and how connectivity determines patterns of activity in the neuronal population. In order to resolve these spatiotemporal patterns of firing, it is necessary to record activity at multiple sites simultaneously. A powerful experimental tool for this purpose is the planar electrode array (PEA), which can be used as a substrate for inherently twodimensional networks such as dissociated cell cultures or organotypic slice cultures. PEAs also allow the possibility of stimulation at selected sites in the network. Using PEAs or similar devices, the network activity of hippocampal slices (Whecler and Novak, 1986; Boppart et al., 1992), the retinal ganglion cell layer (Meister et al., 1991) and spinal cell cultures (Droge et al., 1986) has been investigated.

In this article, we have extended our previous work on the mechanism of synchronized bursting in cultured networks of cortical neurons (Robinson et al., 1993), by using PEAs to study detailed multisite patterns of firing. We examine the factors affecting the velocity, origin, and direction of burst propagation as resolved by multisite recording. In addition, we characterize the refractoriness and propagation of bursts elicited by stimulation through single sites in the PEA. The results provide a 

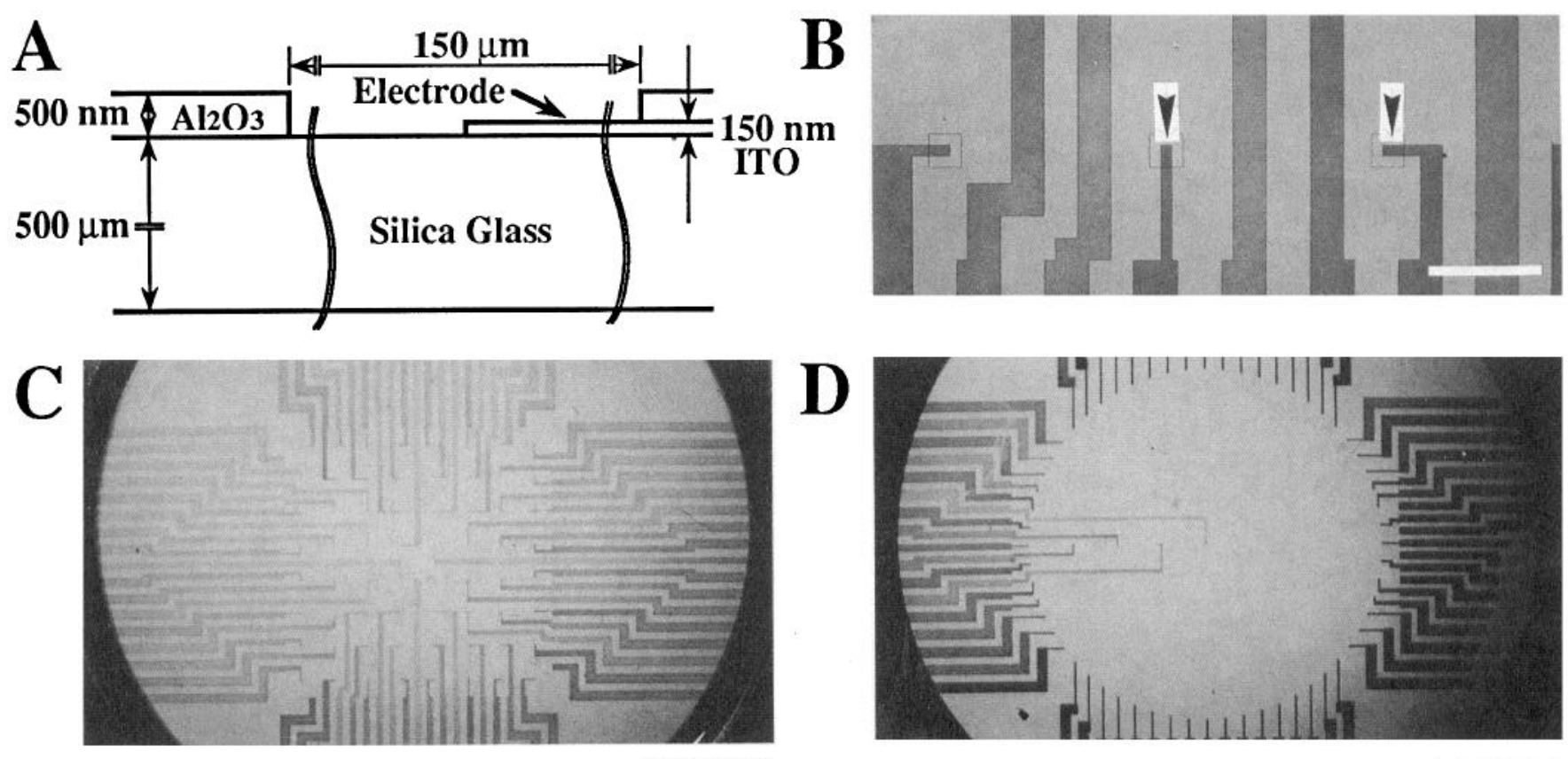

D

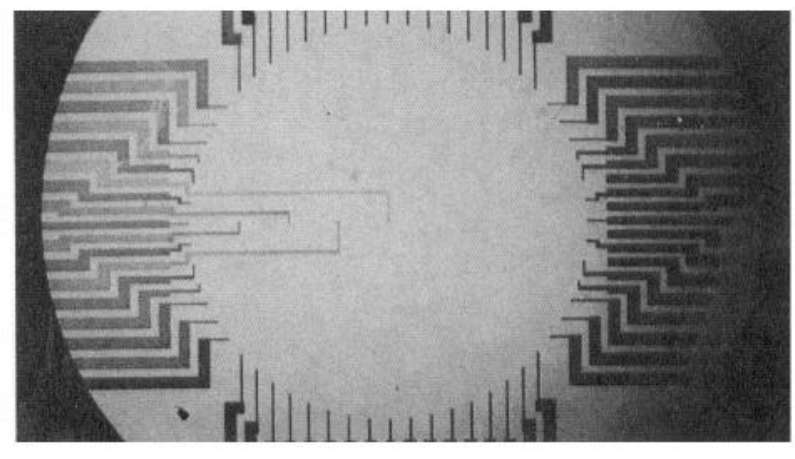

$\mathbf{E}$
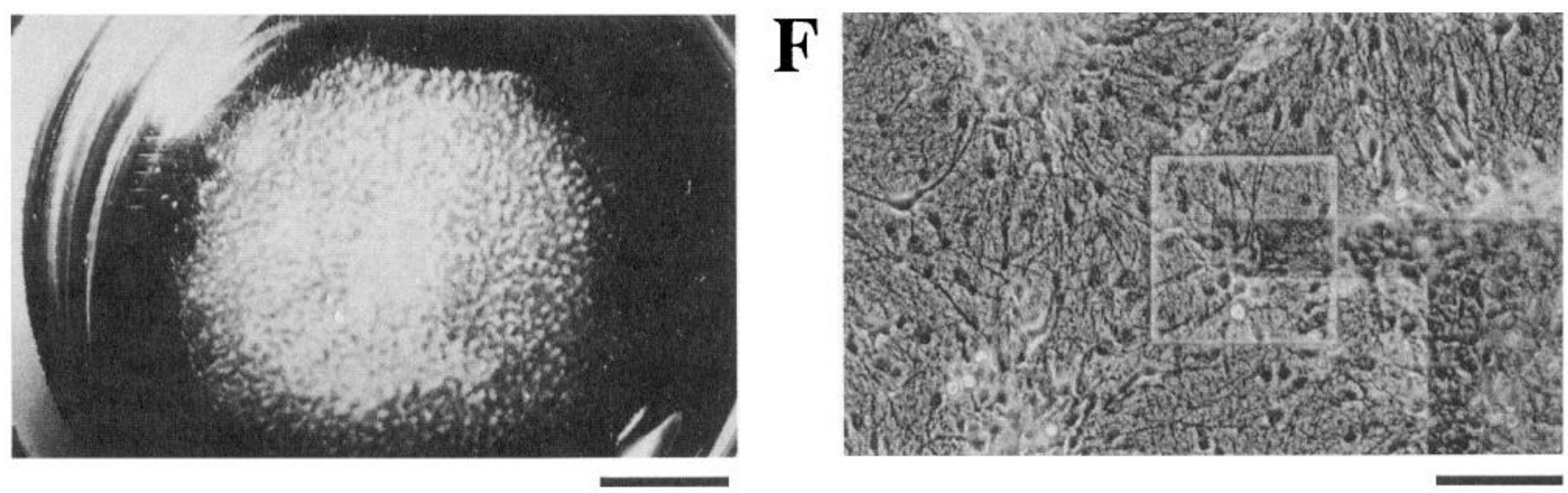

Figure 1. A diagrammatic cross section $(A)$, a closeup of a region of the PEA $(B)$, the layout of two types of PEAs $(C$ and $D)$, and a cultured neuronal network on a PEA 2 weeks after plating $(E$ and $F)$. In $B$, the arrows indicate electrodes. Scale bars represent $500 \mu \mathrm{m}(B), 3 \mathrm{~mm}(C, D$, and $E)$, and $100 \mu \mathrm{m}(F)$.

foundation for a better understanding of the mechanism of synchronized firing and repetitive bursting in cortical neuronal networks.

Some of these results have been presented in abstract form (Maeda et al., 1993; Maeda and Kawana, 1994).

\section{Materials and Methods}

Electrode arrays. Planar electrode arrays (PEAs) were fabricated by a method similar to that described in Jimbo et al. (1993). Briefly, a 150 $\mathrm{nm}$ sputtered film of the transparent conductor indium tin oxide (ITO) was patterned on a $500 \mu \mathrm{m}$ thick silica glass wafer by photolithography, using wet etching in $\mathrm{HCl}$. A $500 \mathrm{~nm}$ insulating layer of $\mathrm{Al}_{2} \mathrm{O}_{3}$ was then applied by evaporation to the whole substrate, but lifted off at the tips of the ITO electrodes $(100 \mu \mathrm{m} \times 50 \mu \mathrm{m})$ by photolithographic patterning. The exposed electrode tips were platinized by electrolytic deposition. Figure $1 A$ illustrates the structure of the PEAs. Two types of PEA were prepared. One comprised 64 electrodes in an $8 \times 8$ grid with $1 \mathrm{~mm}$ between adjacent electrodes (Fig. $1 C$ ), while the other consisted of a $10 \mathrm{~mm}$ diameter circular arrangement of 64 electrodes separated by about $500 \mu \mathrm{m}$ (Fig. $1 D$ ). A network cultured on a PEA is shown in Fig. $1 E$ and $F$.

Cell culture. Cell cultures were prepared from the cerebral cortex of embryonic day 17-18 Wistar rats, dissociated using papain as described in Muramoto et al. (1988). The cells were plated on the PEAs, which were pretreated with laminin and poly-D-lysine followed by a final coating of laminin. The cell density on the PEA was approximately $10^{4}$ cells $/ \mathrm{mm}^{2}$. Without preincubation with laminin before poly-lysine application, cells tended to form small aggregates, apparently due to insufficient attachment of poly-lysine to the substrate. Cultures were treated with $10 \mu \mathrm{M}$ cytosine arabinoside (AraC) at $4 \mathrm{~d}$ in vitro (DIV) for 1 d and maintained in Dulbecco's Modified Eagle's Medium (DMEM) containing $5 \%$ heat-inactivated fetal bovine serum, $5 \%$ heat-inactivated horse serum, $50 \mathrm{U} / \mathrm{ml}$ penicillin, $50 \mu \mathrm{g} / \mathrm{ml}$ streptomycin, and $2.5 \mu \mathrm{g} /$ $\mathrm{ml}$ insulin, in a $90 \%$ air, $10 \% \mathrm{CO}_{2}$, and $\mathrm{H}_{2} \mathrm{O}$-saturated atmosphere at $37^{\circ} \mathrm{C}$. Half of the medium was replaced by fresh medium every 3 or 4 d. For experiments before about 10 DIV, cultures were not treated with AraC.

Electrical measurements and staining. Extracellular recording was conducted at room temperature at 1-4 weeks after plating. Signals from 16 of the 64 electrodes were amplified $10,000 \times$ using a 16-channel amplifier (MEG6100, Nihon Kohden), high-pass filtered at $150 \mathrm{~Hz}$, and recorded on a 16-channel DAT recorder (RD-200, TEAC) at 14-bit resolution, with a sampling frequency of $6 \mathrm{kHz}$ per channel, using appropriate anti-aliasing low-pass filtering. A manual switch array was used to select a subset of 16 of the 64 channels at any one time for recording. The bath solution contained (in mM) $150 \mathrm{NaCl}, 2.8 \mathrm{KCl}, 1 \mathrm{CaCl}_{2}, 10$ $N$-2-hydroxyethylpiperazine- $N^{\prime}$-2-ethane-sulfonate (HEPES)-Na, 10 
glucose, 0.003 glycine, and $0-2 \mathrm{MgCl}_{2}, \mathrm{pH} 7.2$. Whole cell recording was carried out by standard methods (Hamill et al., 1981) using a commercially available amplifier (Axopatch $1 \mathrm{C}$, Axon Instruments). Pipettes were filled with (in $\mathrm{mM}$ ) 145 potassium gluconate $(2,3,4,5,6$-pentahydroxycaproate, $\left.\mathrm{C}_{6} \mathrm{H}_{11} \mathrm{O}_{7} \mathrm{~K}\right), 2 \mathrm{MgCl}_{2}, 10$ HEPES-Na, 10 glucose, 0.1 $\mathrm{CaCl}_{2}, 1$ ATP- $\mathrm{Na}_{2}, 1.1$ ethylene glycol-bis( $\beta$-aminoethyl ether)$N, N, N^{\prime}, N^{\prime}$-tetraacetic acid (EGTA), pH 7.2. To determine the extent of cells' processes and test for gap junction coupling, $1 \mathrm{mg} / \mathrm{ml}$ Lucifer yellow (Aldrich) or $1-4 \mathrm{mg} / \mathrm{ml}$ Neurobiotin (Vector) was included in the patch pipette. Following 5-20 min in whole-cell recording mode and careful withdrawal of the pipette to permit resealing of the membrane, cells were fixed in $4 \%$ paraformaldehyde in phosphate-buffered saline (PBS) for $20 \mathrm{~min}$. Lucifer yellow was then visualized by epifluorescence microscopy. Neurobiotin was visualized by one of two methods. In the first method, a further $30 \mathrm{~min}$ of incubation was carried out in $10 \mu \mathrm{g} / \mathrm{ml}$ streptavidin-fluorescein (Serotec) and $0.2 \%$ Triton $\mathrm{X}$ in PBS, followed by epifluorescence microscopy (Peinado et al., 1993). In the second method, Neurobiotin was visualized by 30 min incubation in $10 \mu \mathrm{g} / \mathrm{ml}$ streptavidin-conjugated horse radish peroxidase (Serotec) and $0.2 \%$ Triton X in PBS, followed by $15 \mathrm{~min}$ of reaction with $0.1 \%$ diaminobenzidine, $0.004 \%$ hydrogen peroxide in phosphate buffer, and observed by bright-field microscopy.

Analysis. Analysis of extracellular signals was conducted after digital transfer of signals from the tape recorder to computer (SparcStation 2, Sun Microsystems). The mean value, $\mu$, and standard deviation, $\sigma$, of the background noise on each recording channel were estimated and only signals whose amplitude was greater or less than $\mu \pm 4.42 \sigma$, respectively, were analyzed as action potentials $(p<0.001)$.

Physical sectioning by ultraviolet laser. Physical sectioning of cultured networks on PEAs was carried out with a pulsed ultraviolet (UV) laser with a peak wavelength of $337 \mathrm{~nm}$ (VSL337ND, Laser Science Inc.), focussed through the epifluorescence illumination port of an inverted microscope (Zeiss Axiovert 25). After sectioning, the preparation was washed with bath solution several times. The width of the laser spot was less than $10 \mu \mathrm{m}$ but it destroyed the network up to about 50 $\mu \mathrm{m}$ from the center of the spot (See Fig. $7 C$ ). During cutting, the power of the laser was carefully controlled using neutral density filters and by varying the pulse rate so as to achieve the smallest possible, visibly complete cut.

\section{Results}

\section{Parallel recording of synchronized bursting}

The electrical activity of cultured networks covering about 100 $\mathrm{mm}^{2}$ in total area was recorded from up to 16 electrodes simultaneously using PEAs, whose layout is shown in Figure 1. The eight channels shown in Figure 1 are selected for large signal to noise ratios after sampling from all 64 channels. Spontaneous bursts of action potentials appeared, when recorded in nominally magnesium-free bath solution to stimulate activity, after 3 or 4 $\mathrm{d}$ in vitro (DIV). The probability of observing spike activity at individual electrodes that was significant relative to the baseline noise variance (see Materials and Methods) increased with time in culture, in parallel with an increase in the amplitude of the extracellularly recorded action potentials.

Figure 2 shows a typical set of signals from a 10 DIV culture. All channels show synchronized bursts lasting for a few hundreds of milliseconds at approximately 10-20 sec intervals. In addition, continuous random firing was observed, as in channel 2 of Figure 2, but at less than $1 \%$ of electrodes that showed activity.

The most striking features of the synchronized burst firing were the stability of the mean burst frequency and the total quiescence of the interburst periods at the vast majority of channels. In fact, the local structure of the spontaneous bursting was more complex than simply periodic, for example, often showing two successive bursts at roughly constant intervals followed by a shift to a different frequency, as seen in Figure 2.

Synchronized bursting could be inhibited by raising the external magnesium concentration, or by perfusing with the
$N$-methyl-D-aspartate (NMDA) receptor antagonist D-2-amino5-phosphonovalerate (APV), or the non-NMDA receptor antagonist 6-cyano-7-nitroquinoxaline-2,3-dione (CNQX), (for example, at $2 \mathrm{mM}, 100 \mu \mathrm{M}$, and $50 \mu \mathrm{M}$, respectively, for $11 \mathrm{DIV}$ cultures). In immature cultures, an intermittent or partial synchronicity of spontaneous firing was often observed, with only some bursts or spikes synchronized at varying subsets of the active electrodes. The same type of partial synchronicity could also be induced by perfusion with low concentrations of APV or CNQX (for example, $20 \mu \mathrm{M}$ APV or $10 \mu \mathrm{M}$ CNQX for 11 DIV cultures) (Maeda et al., 1993b).

\section{Initiation of synchronized bursts}

The bottom panel of Figure 2 shows two successive synchronized hursts at a higher time resolution. The different order of initiation of activity shows that the direction of propagation of spontaneous bursts varies from burst to burst. This suggests that the source or initiation point of spontaneous bursts is not at a fixed locus in the network.

To show this more clearly, the successive delays to initiation of spike activity at each channel were plotted relative to the onset at a single channel. Figure 3 shows the onset delays of nine consecutive bursts relative to channel 8 in the same network as in Figure 2. The continuous variations both in the spread of the latencies and their order is consistent only with an initiation locus, which varies from burst to burst and therefore produces different directions of propagation in the area of the network sampled by the electrode array (Fig. 3, bottom). The average spced of propagation cstimatcd from these results was $-50 \mathrm{~mm} /$ sec. Propagation from the source was not completely smooth, but showed local variations in speed, consistent with the observed variations in cell and projection density.

Synchronous bursts could also be induced in the same network by local stimulation using a current pulse of $100 \mu \mathrm{sec}$ duration at a single electrode in the array, and propagated thence over the whole network. Figure 4 demonstrates the propagation of a burst, at high time resolution, to eight different electrodes after stimulation at points A (left) or B (right) in the network. The order of initiation approximately follows the order of the distance of each electrode from the stimulus site. This result shows that bursts can be initiated from single foci, and that multiple points in the network have the capability to initiate bursts. Using this type of stimulation, it was also possible to lock the phase of periodic bursting to regular stimulating current pulses.

Bursts were evoked locally to the stimulating clcctrodc, sincc a clear threshold of stimulus intensity was observed, and stimulation through some electrodes failed to elicit any burst even with stimulus intensities up to $20 \mathrm{~V}$, implying that excitation did not spread appreciably. The probability of observing such ineffective sites and the stimulation intensity necessary for eliciting bursts both decreased during culture, for example at $30 \mathrm{DIV}$, to over $90 \%$ and less than $1 \mathrm{~V}$, respectively.

Electrical stimulation applied while a burst was still in progress was not capable of prolonging the burst or eliciting a new burst. The reason for this is believed to be that the long-lasting intracellular plateau potential during bursts inactivates inward voltage-dependent ionic currents, thus preventing new burst generation until after a period of recovery (Robinson et al., 1993). Figure 5 shows responses elicited in a 4 DIV culture to repetitive stimulation at various fixed intervals $(5-30 \mathrm{sec})$. Each stimulation consisted of a train of $10 \times 2000 \mathrm{mV}$ pulses separated by $1 \mathrm{msec}$. This procedure revealed a clear refractory period fol- 

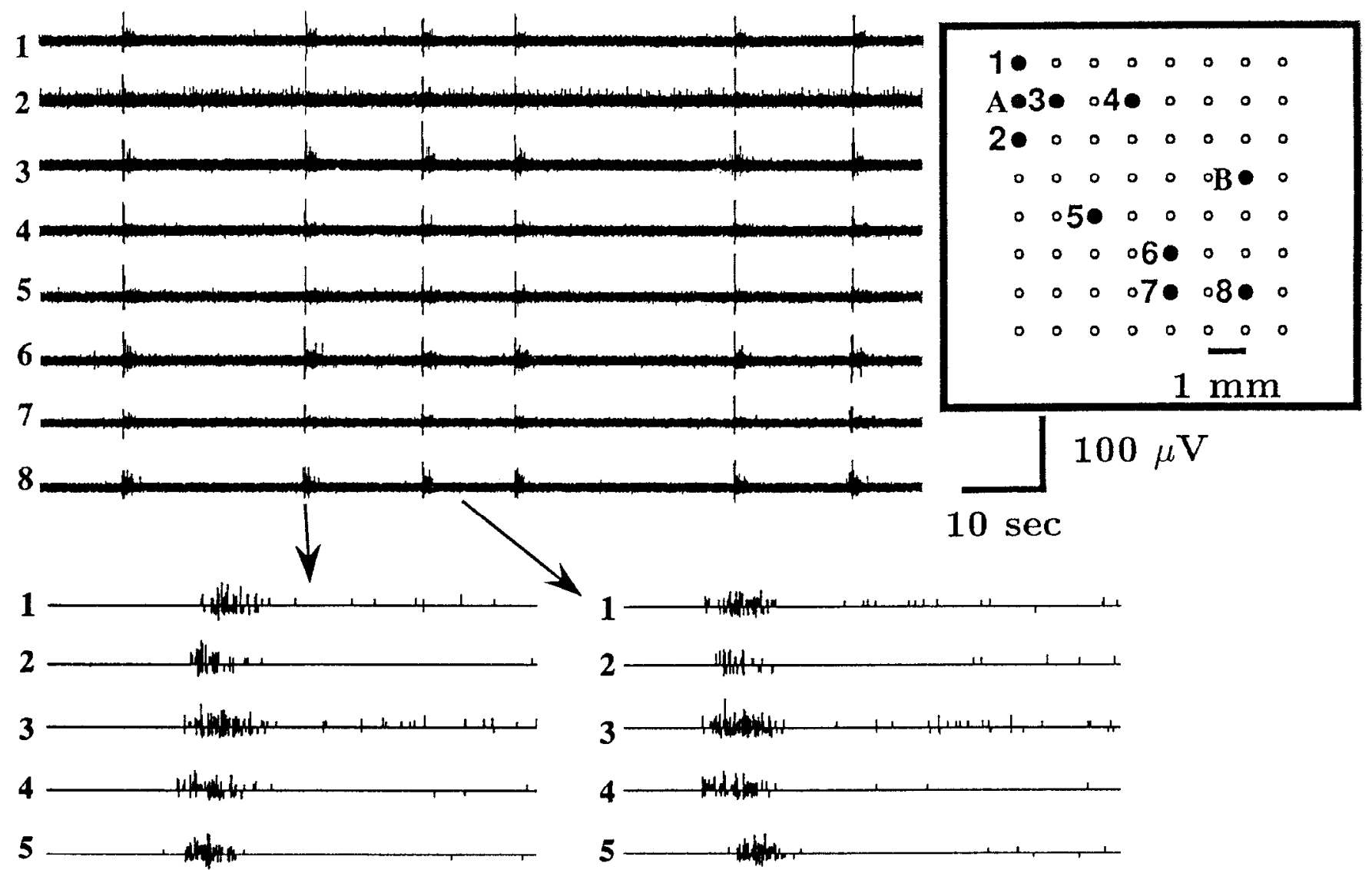

\section{$10 \mathrm{sec}$}

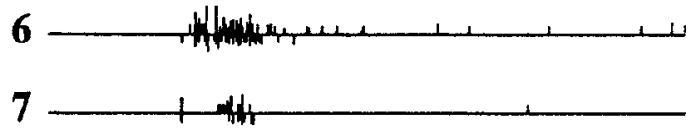

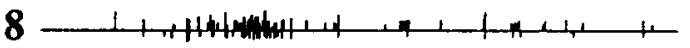

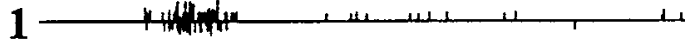

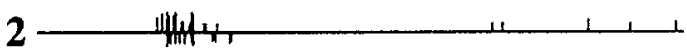
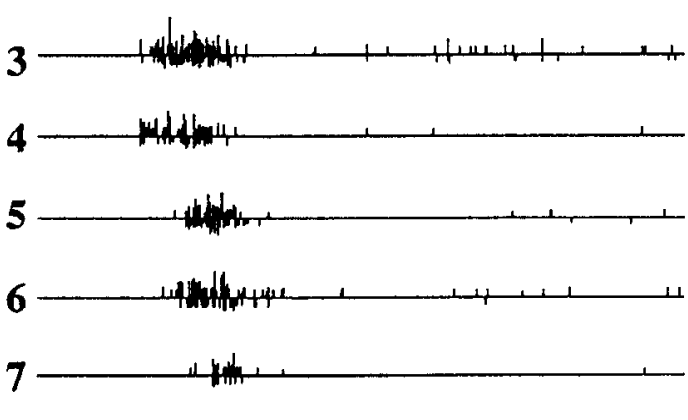

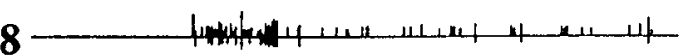

\section{$200 \mathrm{msec}$}

Figure 2. Spontaneous periodic bursts of a cultured network of cortical neurons (10 DIV) recorded at eight different electrodes (top), the physical layout of the electrodes (inset), and two successive synchronized bursts at a higher time resolution (bottom). The estimated baseline noise was removed (see Materials and Methods). Recording was carried out in nominally zero $\left[\mathrm{Mg}^{2+}\right]_{o}$ bath solution. Note the shift in the direction of propagation (order of initiation) of the burst. The data in Figures 3, 4, and 7 are from the same network as used in this figure.

lowing each burst, during which no burst could be induced by electrical stimulation. In this case, the refractory period was more than $5 \mathrm{sec}$ and less than $10 \mathrm{sec}$.

Figure 6 shows a simultaneous recording of extracellular signals through a single site in the PEA and excitatory synaptic currents in a single neuron recorded with whole-cell voltage clamp, during spontaneous bursting and with electrical stimulation at 1 and $2 \mathrm{sec}$ intervals. The stimulation electrode was located at about $10 \mathrm{~mm}$ from the recording PEA electrode and voltage-clamped neuron. The synaptic currents elicited by $2 \mathrm{sec}$ interval stimulation were reduced by about $25 \%$ relative to the spontaneous synaptic currents. One second interval stimulation revealed both absolute refractory periods, during which neither signals from PEA electrodes nor synaptic currents could be detected, and relative refractory periods, during which a reduced number of spikes was observed through the PEA, associated with faster-decaying, smaller-amplitude synaptic currents.

\section{Propagation of bursts}

To investigate whether synchronized activity is due to propagation of electrical excitation or diffusion of an extracellular chemical factor, synchronously bursting networks were physically sectioned using a UV laser. In all 23 preparations subjected to this procedure, synchronous bursting also separated after sectioning into different frequencies and phases in each part. No consistent change in burst frequency was observed in isolated areas from that before sectioning. Figure $7 \mathrm{~A}$ shows a typical example, which is from the same culture as shown in Figure 2. No bursts propagate across the boundary between the two regions. In some experiments, the network was sectioned into three or four parts, and independent spontaneous bursting was observed in each part. Thus, propagation of activity does not appear to be mediated by diffusion of an extracellular substance, but to occur through the network of interneuronal projections.

Partial sectioning resulted in an increased propagation delay (Fig. 7B). The frequency of spontaneous bursts did not change significantly, but the propagation delay between, e.g., electrodes 4 and 7 increased from about 100 to about $200 \mathrm{msec}$. This result indicates that the density of projections between areas is a major determinant of the propagation velocity.

When electrical stimulation was applied from a single elec- 
Figure 3. The delay of burst onset at multiple sites relative to that recorded at channel 8 in Figure 2. Nine consecutive bursts over a period of about 10 sec were analyzed. The relative delay was estimated as the time between the first spike at channel 8 and the first spike at each respective channel. The order of initiation indicates the direction of burst propagation---for example, the second, fourth, and eighth bursts propagate from electrode 1 towards electrode 8 while the sixth, seventh, and ninth bursts propagate in the opposite direction. The numbers in the upper panel indicate how the bursts were classified into four different approximate directions of propagation, as shown at the bottom.
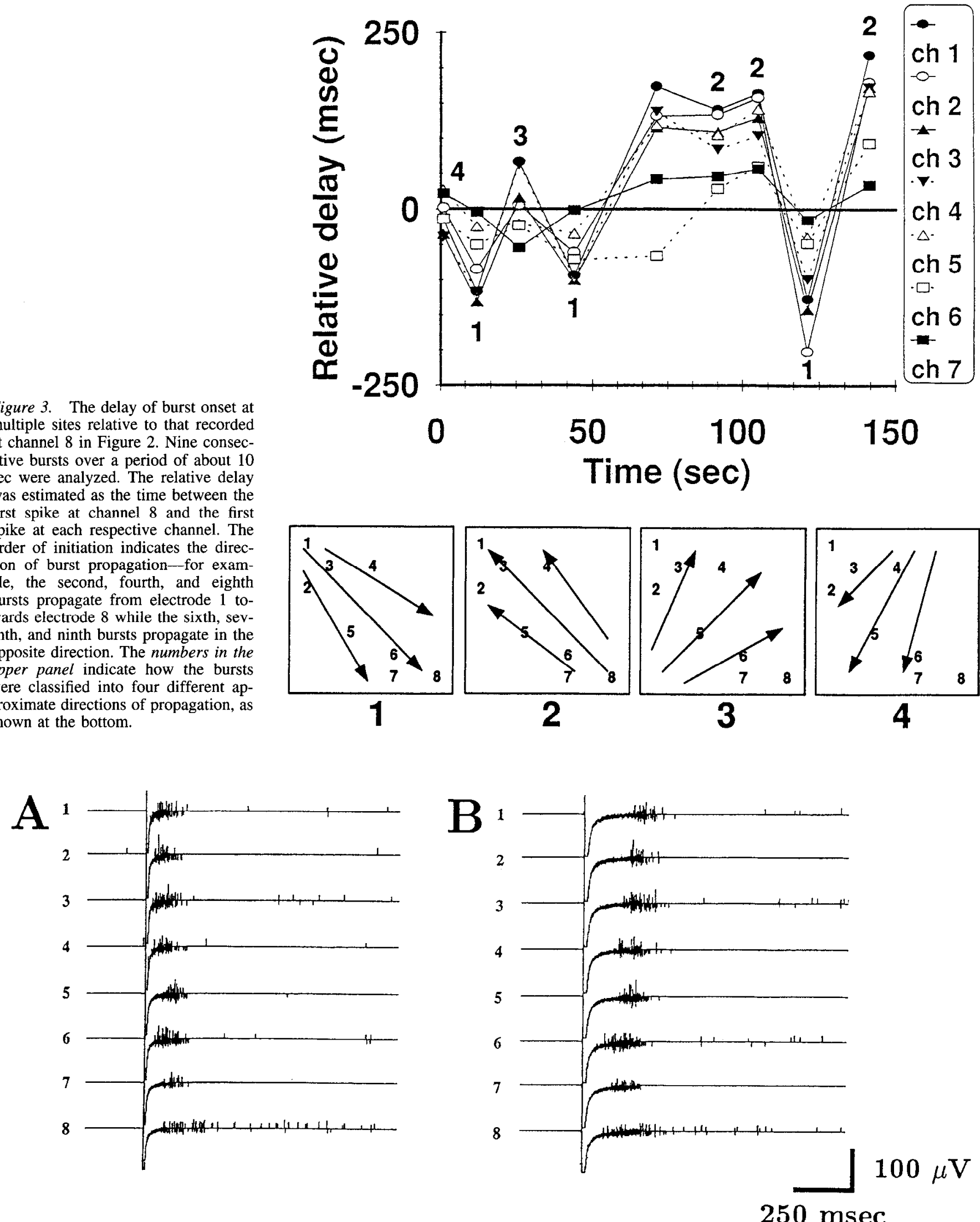

Figure 4. Synchronous bursts in the same preparation as in Figure 2, elicited by electrical stimulation from electrodes A and B in Figure 2 and recorded from electrodes $1-8$. The early parts of bursts overlap with the stimulation artefact. In $A$, the evoked bursts propagate from electrode $A$ towards electrode 8 , while in $B$, the evoked burst reaches electrode 1 later than it reaches electrode 8 . The difference in relaxation time of the stimulus artefact reflects a difference in the impedance of electrodes $\mathrm{A}$ and $\mathrm{B}$ and a difference in the stimulation intensities (1500 and $1800 \mathrm{mV}$, respectively). 

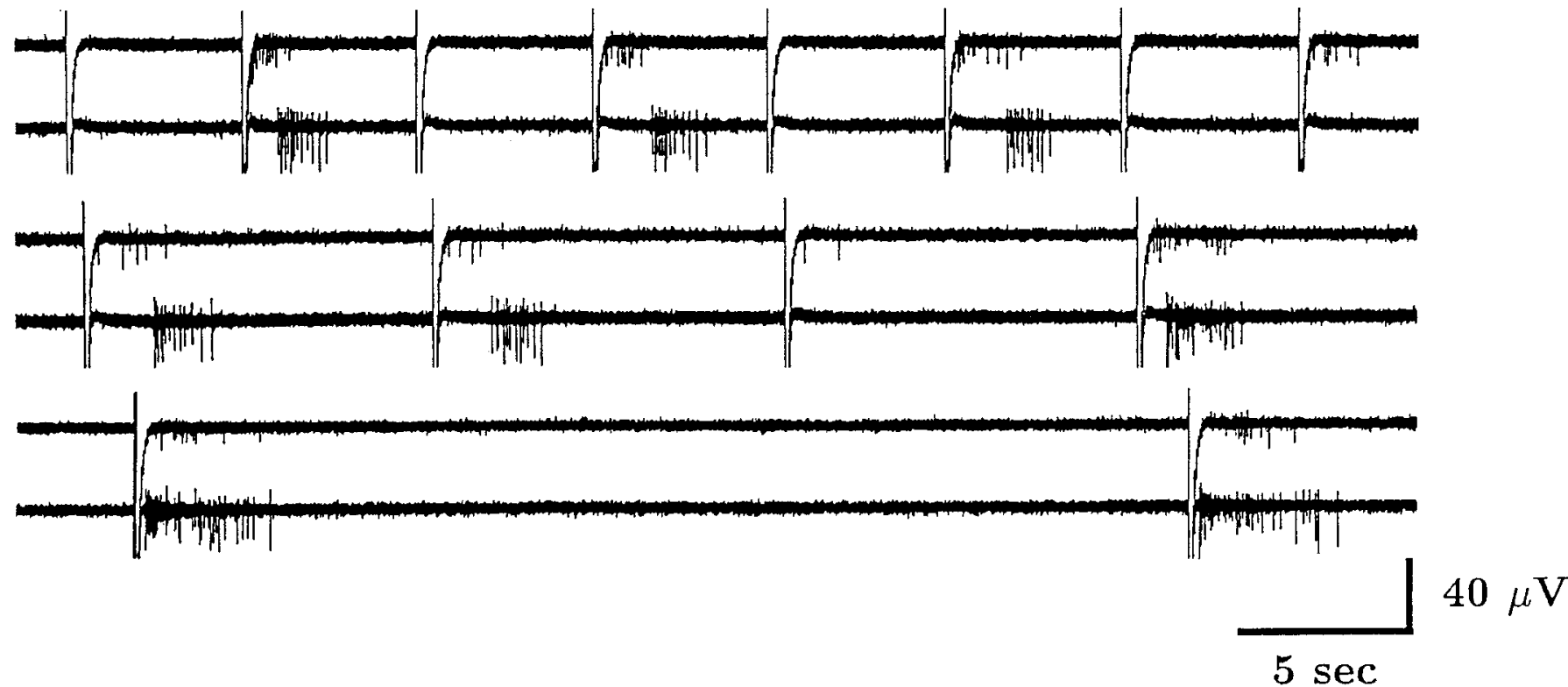

Figure 5. Refractoriness of bursts. Each pair of traces shows the signals from two electrodes in a $4 \mathrm{DIV}$ culture in zero [Mg $\left.{ }^{2+}\right]_{v}$. The recording electrodes were located $1.5 \mathrm{~mm}$ (top traces) and $8 \mathrm{~mm}$ (bottom traces) from the stimulation electrode, corresponding to locations E1 and E3, respectively, in Figure 8. The stimulus electrode was at position E0 in Figure 8. Bursts were elicited by electrical stimulation at 5 (top), 10 (middle), and 30 (bottom) sec intervals. Spontaneous bursts were observed at about $30 \mathrm{sec}$ intervals (not shown). At 5 sec intervals, every other stimulation falls in a refractory period, while at $10 \mathrm{sec}$ intervals, a refractory period is only encountered occasionally (third stimulus). At 30 sec intervals, no failures could be recorded.

A

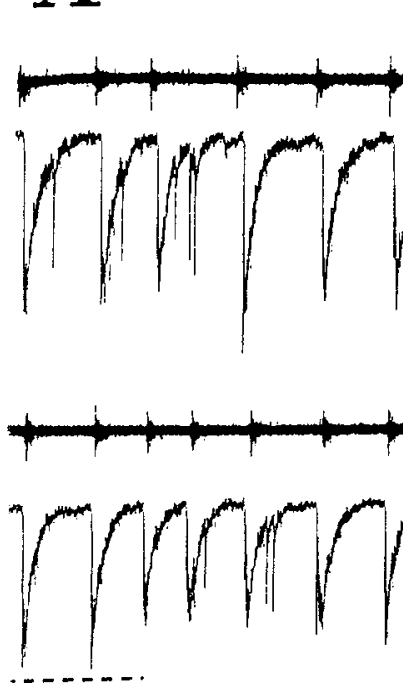

B
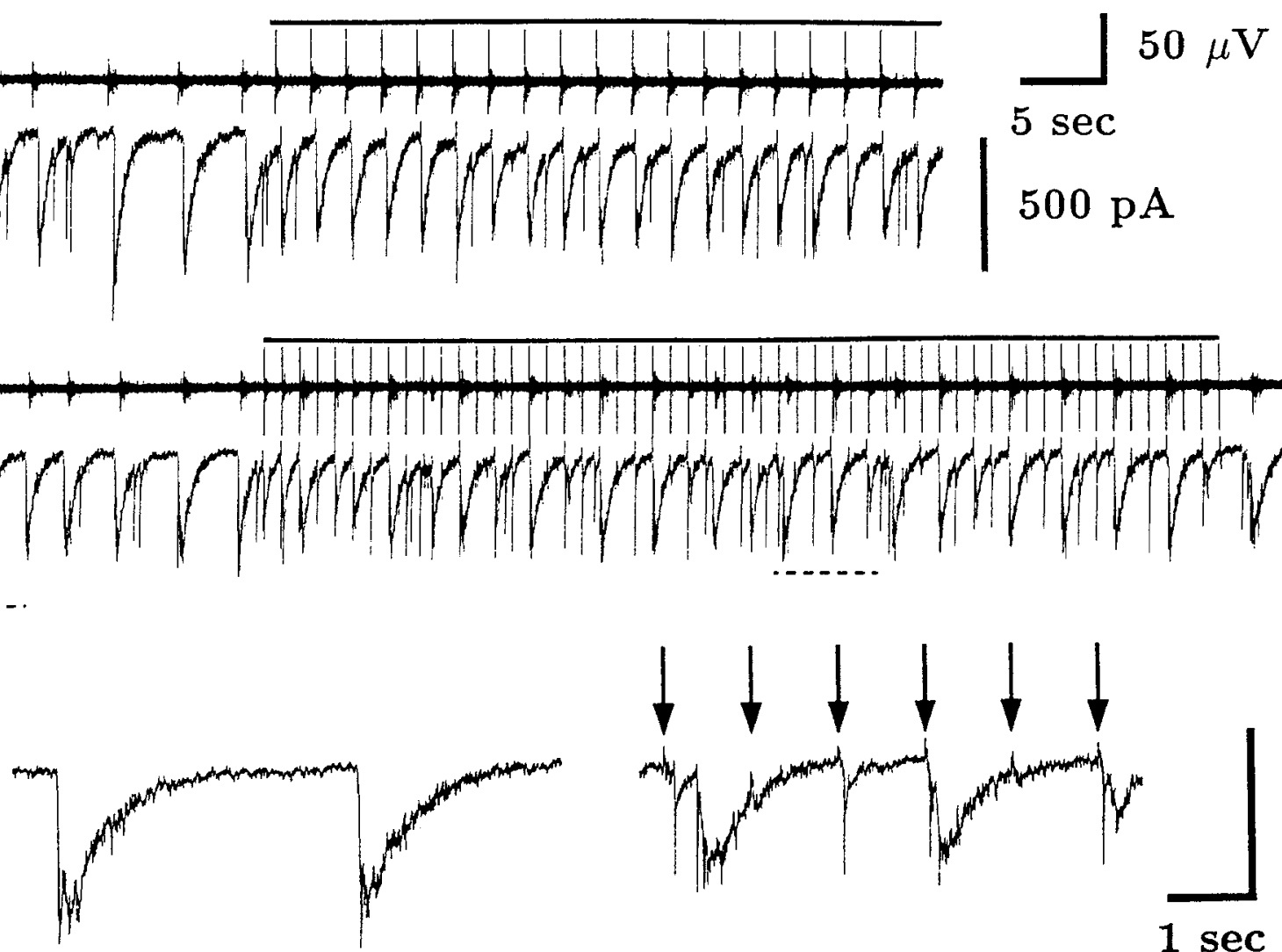

500 pA

Figure 6. Simultaneous recordings from a PEA electrode (A, upper traces) and a whole-cell patch pipette in voltage-clamp mode (holding potential $-50 \mathrm{mV}$, lower traces). Bursts were accompanied by synaptic currents both in spontaneous firing, and with electrical stimulation through the PEA at 2 and $1 \mathrm{sec}$ intervals. Periods of stimulation are indicated by bars. $B$, Higher time resolution traces during the periods indicated by the dotted lines in A. Arrows indicate the times at which stimulation was applied. 


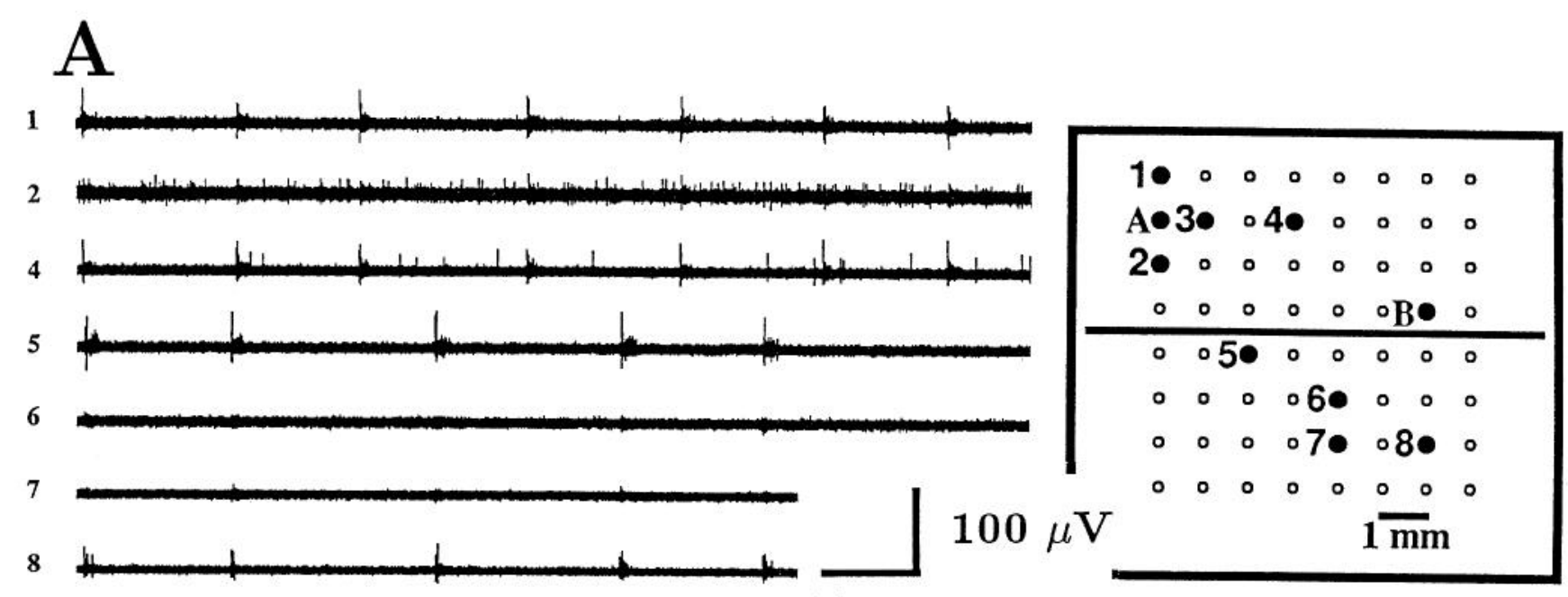

\section{$10 \mathrm{sec}$}
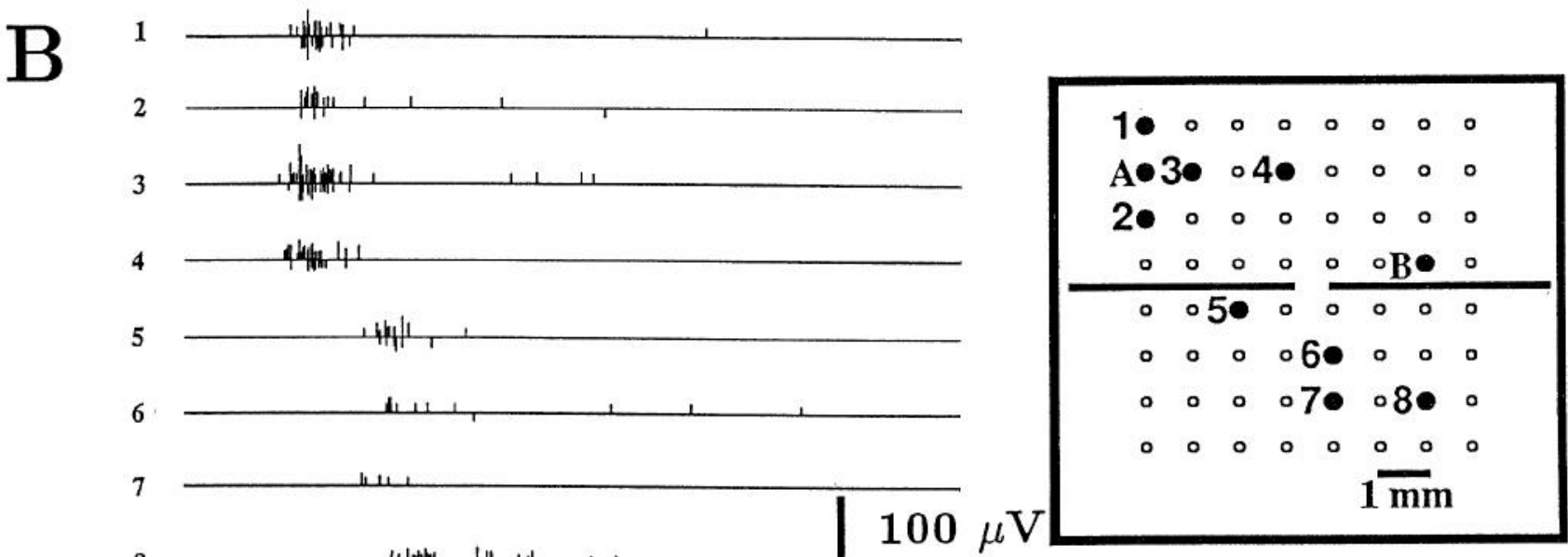

\section{$250 \mathrm{msec}$}

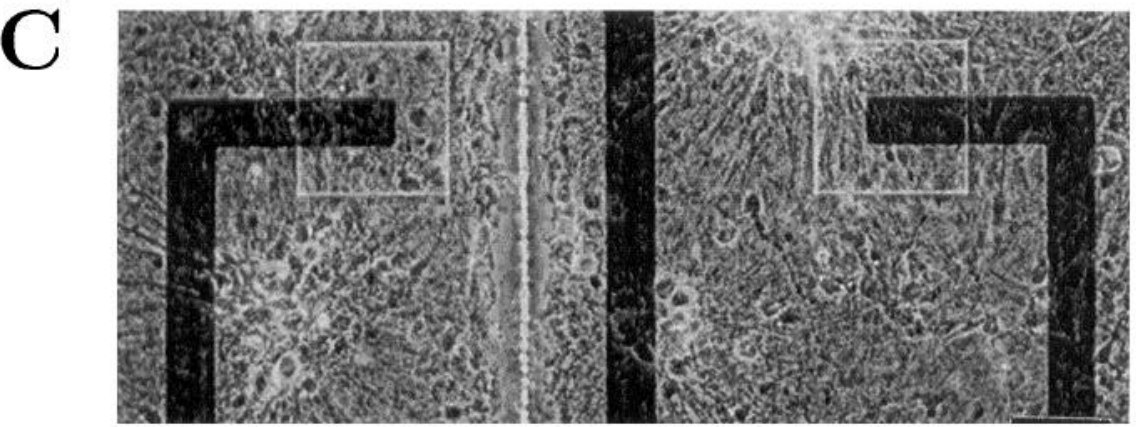

Figure 7. The effects of physical separation of synchronously bursting networks into several sections using a UV laser. A, After sectioning, synchronous bursting also separated into different frequencies and phases in each part. The position of sectioning is indicated by the black line across the inset showing the electrode array. B, Spontaneous bursts in a partially sectioned network, as indicated by the line across the inset. After partial sectioning, bursts propagated across the cut with a much increased delay. Bursts recorded in the same network before cutting are shown in A. C, A partial view of a sectioned cultured network. Scale bar represents $100 \mu \mathrm{m}$.

trode to a laser-sectioned network, it evoked synchronous bursts only within the laser-delimited region in which the electrode was located. Even if the site of the stimulation electrode was near (about $120 \mu \mathrm{m}$ ) the line of cutting, no excitation propagated across the boundary (not shown). This result is further evidence that electrical stimulation through a single PEA electrode is lo- calized to its immediate environment. Furthermore, the initiation of independent bursting in laser-isolated areas and the ability to evoke bursts within each area indicates that in fairly mature cultured networks of cortical neurons ( $>$ about 6 DIV), almost any site is a potential burst source.

As shown in Figure 5, the latency of evoked bursts, which 

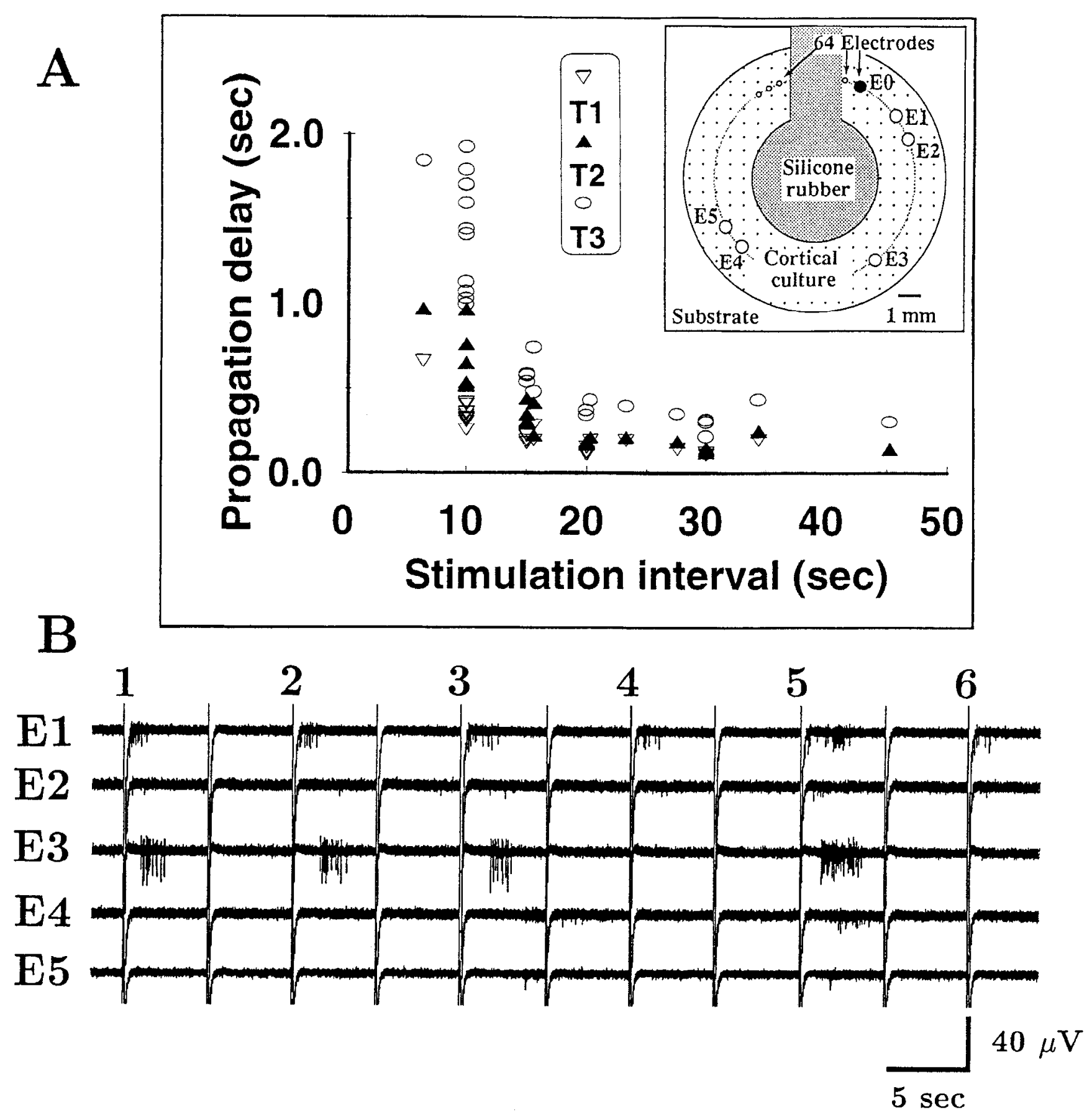

Figure 8. Measurement of burst propagation. A, The time required for bursts to propagate between two electrodes as a function of the stimulation interval in a day 4 culture at nominally $0\left[\mathrm{Mg}^{2+}\right]_{0}$, and the circular layout of the electrodes (inset). The cells were confined to the ring surrounding the central region of silicone rubber. Stimulation was applied at E0 and the evoked activity recorded at E1,E2, and E3. TI, T2, and $T 3$ indicate the propagation delays recorded at E1, E2 and E3, respectively. B, Partial propagation of evoked bursts. Signals recorded at E1 through E5 from the same preparation as shown in A. The third and fifth evoked bursts propagated to E5, the first and second bursts only to E3, while the fourth and last bursts failed to reach $\mathrm{E} 3$.

reflects the time of propagation from the stimulation electrode to the recording electrode, varies with the stimulation interval. This was demonstrated more clearly by plotting the relationship between the onset time of bursts elicited by focal stimulation and the stimulation interval in a day 4 culture (Fig. 8), plated on a circularly-arranged PEA in order to confine propagation to a single dimension. This revealed a consistent and marked slowing of propagation speed with shorter stimulation intervals. Moreover, this was accompanied by a limitation of the extent of propagation. Repetitive stimulation at intervals close to the refractory period (for example, at $10 \mathrm{sec}$ intervals in a day $4 \mathrm{cul}$ ture) sometimes elicited bursts with only partial propagation (Fig. 8). These results reflect the process of recovery from inactivation processes.

It is possible that, in addition to the excitatory synaptic projections between neurons (see Fig. 6 and Robinson et al., 1993), a significant role is played hy passive propagation of excitation through gap junctions between neurons. In order to examine this 


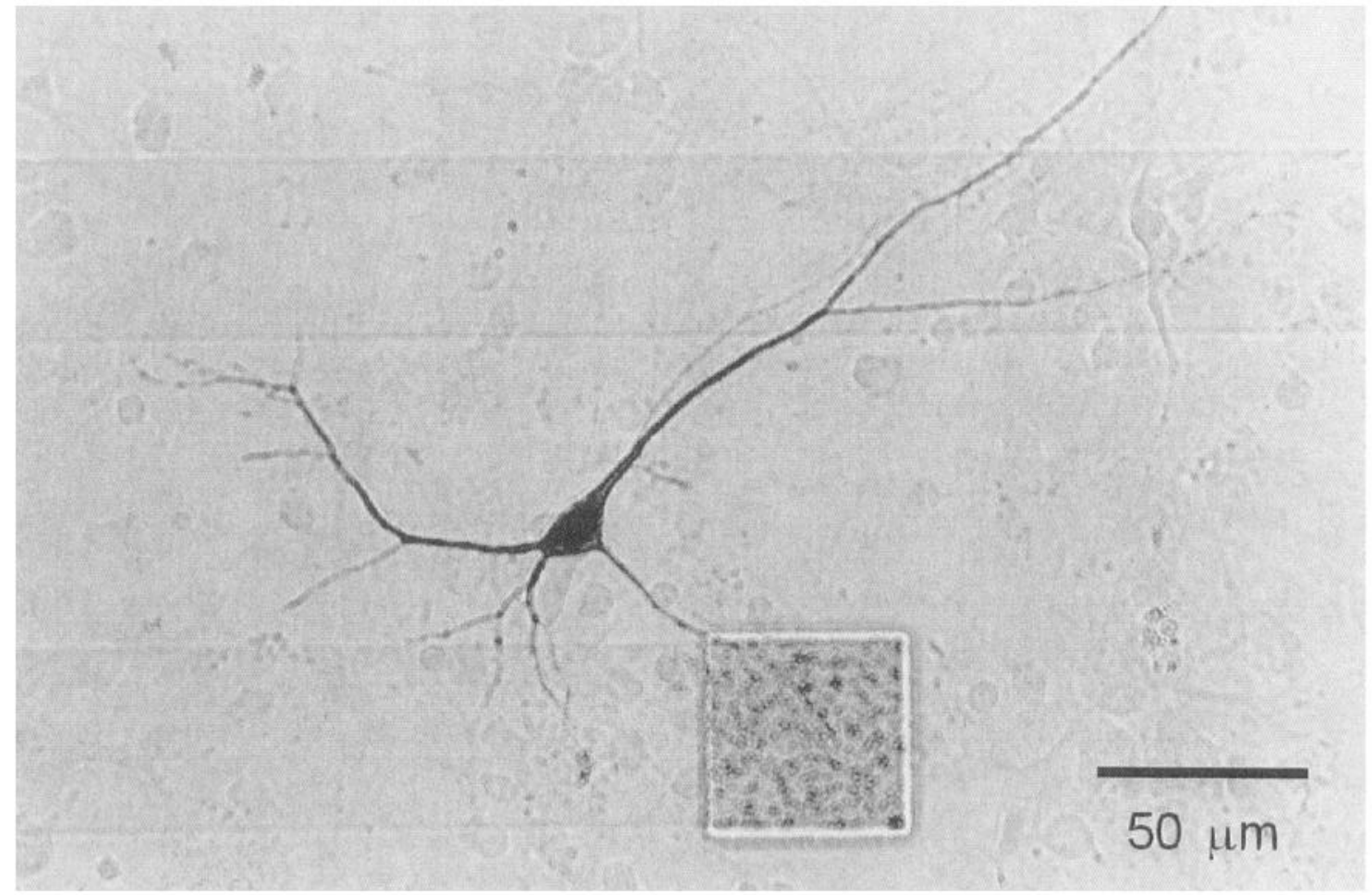

Figure 9. Bright-field photomicrograph of a region of 11 DIV culture. A single pyramidal neuron was filled with Neurobiotin through a wholecell patch pipette and visualized using avidin-conjugated horseradish peroxidase. The granular gray square is an electrode terminal coated with platinum black. No detectable spread of Neurobiotin to other cells is seen, despite multiple points of contact.

possibility, randomly selected cells were filled with the fluorescent dye Lucifer yellow or with Neurobiotin, which were incorporated in the patch pipette during whole-cell recording (see Materials and Methods). Three cells at 6 DIV, one cell at 11 DIV, three cells at 12 DIV, four cells at 15 DIV, and three cells at 18 DIV were filled with Lucifer yellow, but in only one case (at 12 DIV) of the 14 recordings was dye coupling to an adjacent neuron observed. Notably, the soma of this cell was touching the soma of the patch-clamped cell in a small clump of neurons. Nine cells at 11 DIV, five cells at 12 DIV, and two cells at 18 DIV were filled with the smaller tracer molecule, Neurobiotin. No dye coupling was observed in any of these cells. Figure 9 shows a Neurobiotin-filled cell in contact with another cell at 11 DIV, but without observable coupling of the marker molecule. From the present experiments, we can not rule out the possibility that there are significant numbers of Lucifer yellow or Neurobiotin-permeable gap junctions in very dense cultures that were often used in this study, since such cultures prevented adequate whole-cell patch-clamp recording. However, since synchronized bursting was also observed in cultures of uniform density as low as that in which dye filling was carried out, we conclude that excitation can propagate through the network during synchronized bursting, by a mechanism that does not involve Neurobiotin or Lucifer yellow permeable gap junctions amongst the great majority of neurons. The dye-filling experiments also permitted us to map the extent of the processes of neurons in the culture. The visualized maximum extent of cells' processes did not vary significantly between 6 and $18 \mathrm{DIV}$, with an average of about $250 \mu \mathrm{m}$ from the cell body. However, two cells at 11 DIV showed extremely fine, single projections that extended more than $500 \mu \mathrm{m}$, while one cell at 18 DIV extended $390 \mu \mathrm{m}$ from the cell body.

\section{Developmental changes in network activity}

Both spontaneous bursting activity and evoked firing of cultured networks of cortical neurons changed during culture, presumably reflecting the outgrowth and elaboration of cell processes and the maturation of synapses. In nominally $\mathrm{Mg}$-free recording conditions, spontaneous bursts begin to appear at 3 or 4 DIV, and the frequency of bursts increases with further time in culture, to approximately $0.01-0.03 \mathrm{~Hz}$ at $4 \mathrm{DIV}, 0.1-0.2 \mathrm{~Hz}$ at $14 \mathrm{DIV}$, and $0.3-0.5 \mathrm{~Hz}$ at $30 \mathrm{DIV}$. This spontaneous activity was sensitive to external $\left[\mathrm{Mg}^{2+}\right]_{o}$. With higher $\left[\mathrm{Mg}^{2+}\right]_{o}$, a lower frequency of spontaneous bursts was observed, and above a critical concentration, spontaneous bursting ceased altogether. The sensitivity to extracellular $\mathrm{Mg}^{2+}$ decreased during culture. Before 7 DIV, spontaneous bursts disappeared at $100 \mu \mathrm{M}\left[\mathrm{Mg}^{2+}\right]_{o}$, while in cultures older than $30 \mathrm{DIV}$, bursting was maintained even at $2 \mathrm{mM}\left[\mathrm{Mg}^{2+}\right]_{o}$.

This is illustrated in Figure 10, which shows spontaneous and evoked activity in 26 and 9 DIV cultures, recorded in $2 \mathrm{~mm}$ and $100 \mu \mathrm{M}\left[\mathrm{Mg}^{2+}\right]_{o}$, respectively. Under these conditions, the period of spontaneous bursts was about $10 \mathrm{sec}$ in both cultures. In the mature culture (26 DIV), stimuli at $1 \mathrm{sec}$ intervals each evoked a burst, but of shorter duration and fewer spikes than at longer stimulus intervals. Immature cultures, however, such as the 9 DIV culture in Figure 10, showed much longer absolute refractory periods of about $3 \mathrm{sec}$, in which no burst could be evoked even with an increased intensity of stimulation, as well as longer latencies of evoked bursts (slower propagation velocity). More- 

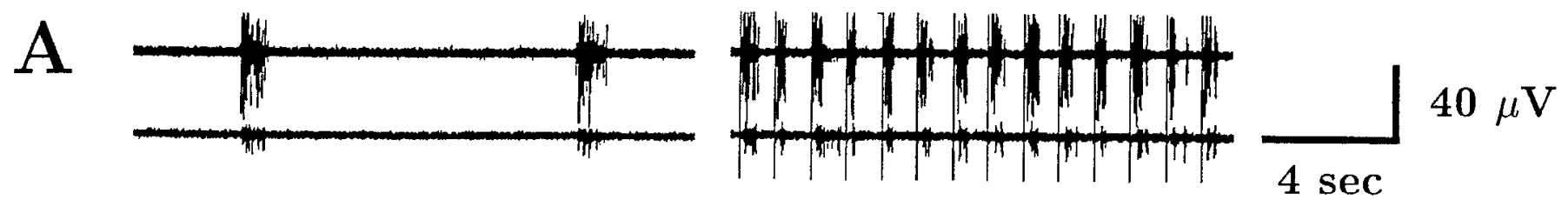

B
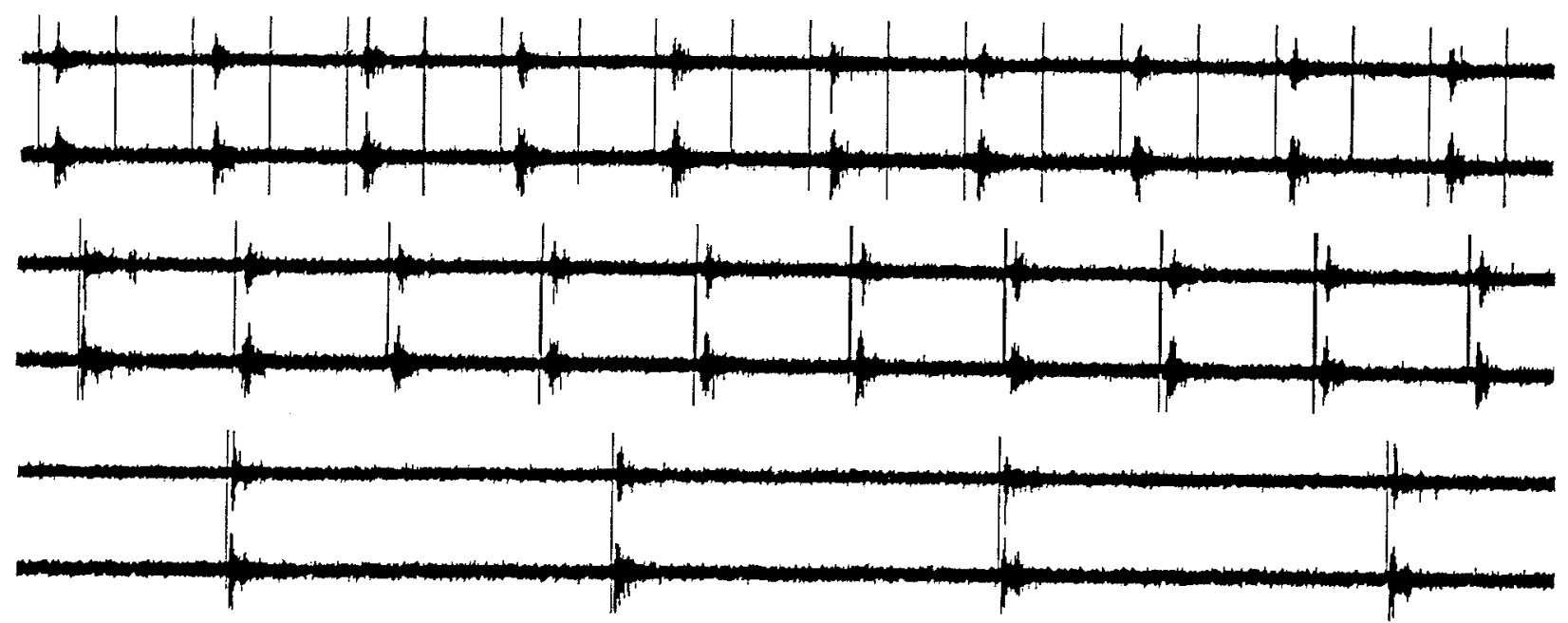

Figure 10. Developmental changes in synchronized bursting. A, Spontaneous bursts (left) and bursts evoked by 1 sec interval stimulation (right) in a 26-d-old culture. B, 2 (top), 4 (middle), and $10 \mathrm{sec}$ interval stimulation (bottom) in a 9-d-old culture. Records show a pair of signals recorded simultaneously at two electrodes in $2 \mathrm{mM}(A)$ and $100 \mu \mathrm{M}(B)\left[\mathrm{Mg}^{2+}\right]_{o}$ respectively. Note the differences in refractoriness, latency, and number of spikes per burst.

over, a 4 DIV culture in nominally zero $\left[\mathrm{Mg}^{2+}\right]_{o}$ solution (see Fig. 5) showed not only absolute refractory periods of about 5 sec, but also relative refractory periods lasting a further 5-15 sec during which bursts were evoked, but propagated only within a subregion of the network.

\section{Discussion}

We have used planar electrode arrays as multisite recording and stimulation devices to study the mechanism of generation and propagation of spontaneous synchronized bursting in cultured networks of cortical neurons. Both extracellular spike signals and stimulation were well-localized to individual electrodes. In recording, "cross-talk," the simultaneous detection of the same spike on more than one electrode, was never observed under the present experimental circumstances, and the number of spikes in each PEA-recorded burst usually corresponded to the number expected from a small number of neurons (1-10) as judged from intracellular recordings. Stimulation, likewise, was restricted to the immediate vicinity of the electrode, because the stimulating electrode current could not produce excitation across a laser sectioning boundary in parts of the network closer than the nearest electrode, as shown in Figure 7. Thus, the use of PEAs allowed an accurate spatial map of activity to be resolved, as well as providing a means of spatially patterned stimulation.

The probability of observing significant spikes at PEA electrodes increased with days in culture as the amplitude of the recorded spikes increased. This was accompanied by a decrease in the threshold stimulus intensity for eliciting bursts. These observations suggest a progressive increase in the degree of electrical coupling between electrodes and overlying neurons, in addition to rising expression of the voltage-dependent inward channels (sodium and calcium) underlying the spike (see McCormick and Prince, 1987).
The cultured networks of dissociated cortical neurons used in this study consist of a sheet of cells of fairly uniform density. Furthermore, the recordings with Lucifer yellow revealed an average maximum extent of neuronal projections of approximately $200 \mu \mathrm{m}$ from the cell body, compared to a network approximately $1 \mathrm{~cm}$ in diameter. Therefore, the network may be considered, in a simplified way, as a collection of multiple overlapped local circuits, forming a two-dimensional excitable medium. This idea is supported by our finding that propagation of activity occurs sequentially from electrode to electrode, as each local group of neurons "charges up" its neighboring, nonrefractory areas, rather than in a random sequence of regions as would be expected if the length of projections were on the same scale as the diameter of the network.

The slow, directional propagation revealed here by PEA recording means that globally synchronized periodic bursts must occur by periodic generation of burst excitation in a part of the network, followed by propagation outwards over the whole network. We found that successive burst events originate in different regions of the network, so that the direction of burst propagation is constantly shifting. In addition, independent burst activity was seen simultaneously in both laser-isolated areas of the network, and network bursting could be initiated by focal stimulation at multiple points in the network. Thus, multiple regions in the network can act as the source of bursts. Robinson et al. (1993) reported that no pacemaker potential is seen intracellularly during periodic bursts, nor any progressive increase of excitability or decrease of conductance during interburst quiescent periods. In the same preparation, desynchronized nonburst firing and partially synchronized bursts have also been observed in special cases, at early stages ( 3 or 4 DIV) of culture (Kamioka et al., 1992), in moderate concentrations of $\mathrm{Mg}^{2+}$, or at APV concentrations that inhibit burst generation but do not complete- 
ly suppress electrical activity (Maeda et al., 1993b), or in cultured networks of reduced area (Maeda et al., 1993a). These results suggest that the periodic bursts are not controlled by specific pacemaker cells but rather are produced by spatial and temporal summation of a continuous random background of synaptic inputs, including "miniature" spontaneous synaptic events.

External magnesium produces inhibition of transmitter release at nerve terminals (Douglas, 1968) and a voltage-dependent block of NMDA receptor channels (Mayer et al., 1984; Nowak et al., 1984), which can explain the decrease in spontaneous burst frequency with increasing $\left[\mathrm{Mg}^{2+}\right]_{o}$. Spontaneous bursts in young cultures in low $\left[\mathrm{Mg}^{2+}\right]_{\sigma}$ solution had a similar frequency to bursts in older cultures in higher $\left[\mathrm{Mg}^{2 !}\right]_{o}$, suggesting that the probability of suprathreshold background synaptic input to a single neuron is nearly equal. However, stimulation experiments revealed fundamentally different characteristics in early and late cultured networks (see Results and Figs. 8 and 10), such as a much longer refractory period at early stages. This suggests that the frequency of synchronized spontaneous bursts is determined not only by the probability of burst generation but also by the probability of successful propagation over the network, both of which factors vary with the number of synapses per cell, the synaptic density and state of maturation, the degree of process extension, and cell excitability. When the probability of burst propagation is low therefore there are abortive burst initiation events that fail to spread beyond their immediate locality, the smallest of which are simply isolated action potentials. Such activity was often seen in immature cultures or in the presence of magnesium or APV.

The early part of the refractory period may be explained in part by residual voltage- and calcium-dependent inactivation of inward current mechanisms in the neurons. However, a more significant source of the observed longer lasting refractoriness is likely to be synaptic depletion of the excitatory transmitter, glutamate, following the dense volley of spikes in each burst, which has recently been shown to recover with a time constant of about $10 \mathrm{sec}$ (Stevens and Tsujimoto, 1995). This would be consistent with the shorter duration and slower velocity of propagation of elicited bursts with stimulation at shorter intervals. An additional contributing factor is likely to be presynaptic inhibition by metabotropic glutamate receptor activation, as a result of extracellular glutamate accumulation (Forsythe and Clements, 1990; Baskys and Malenka, 1991).

The net efficacy of synaptic connections between neurons influenced not only the probability that bursting of neurons in one region could spread to other regions, but also the propagation velocity of signals. This was demonstrated directly in the experiments shown in Figures 7 and 8, which imply that the timing of this type of electrical activity can be controlled by modifications in synaptic efficacy. The observed burst propagation velocities are one to two orders of magnitude smaller than conduction velocities in the smallest unmyelinated nerve fibers (about $1 \mathrm{~m} / \mathrm{sec}$, see Nicholls et al., 1992) and two to three orders faster than the propagation velocity of calcium waves from astrocyte to astrocyte in culture (Nedergaard, 1994). This is also consistent with the structure of the network that comprises multiple overlapping local circuits, as discussed above. Propagation then entails successive integration delays as each local region of cells is charged up to firing threshold. We concluded that propagation is mediated essentially through synaptic projections alone, since ncither gap junctions nor extraccllular diffusion of an excitatory factor appear to be involved. Both the frequency and the propagation velocity of spontaneous periodic bursting seem to be determined by the degree of connectivity of the network, and by the balance between the level of distributed excitation and recovery processes, including the turnover of glutamate.

The same kind of synchronized activity, or the calcium transients associated with it, have already been reported in cultures of cortical neurons (Kuroda et al., 1992; Murphy et al., 1992; Robinson et al., 1992, 1993). In this article, however, we have been able to provide detailed evidence as to the mechanisms of initiation and propagation of synchronized bursts, using parallel recording and stimulation through PEAs. The same type of activity is present in a wide variety of related and different preparations. Charlety et al. (1995) have recently reported identical synchronized bursting in cultured slices of developing rat neocortex, recorded in normal culture medium at $37^{\circ} \mathrm{C}$. In acutely isolated slices of developing cortex, Yuste et al. (1992), have reported that nonsynaptic communication through gap junctions at a certain phase during cortical development defines discrete multicellular domains of synchronized intracellular calcium elevation. In the present study of cultured networks of cortical neurons, however, synchronized activity propagates evenly over the whole network, and gap junctions do not appear to play a primary role in burst propagation at least at 11-12 DIV. These differences could result from the greater degree of focussing or segregation of local connections in acutely isolated slices, or from the higher density of neurons, both of which factors might trigger the formation of gap junction-connected domains of neurons.

The same type of propagating synchronized firing appears to occur in vivo in rat hippocampus (Buzsáki et al., 1992), cat neocortex (Steriade et al., 1993), and where there is a continuous sheet of locally projecting neurons, as in the developing cortex or the in vitro retina (see Shatz, 1990; Meister et al., 1991). It appears to be a stable mode of firing towards which these neuronal networks "relax" following perturbations, for example, by electrical stimulation or by drugs. Moreover, the synchronous bursting can undergo long-lasting changes that apparently involve plasticity of neuronal connections in both the rat hippocampus in vivo (Buzsáki et al., 1992) and in cultured cortical networks (Maeda et al., 1994). The functions of such firing are thus of very general significance in the nervous system, and may include population-wide signaling, refinement of the specificity of point-to-point connections between topographically organized "maps" of neurons, or binding of local circuits into distinct, synchronously firing assemblies such as the cortical column. Future studies will address the role of this mode of firing in shaping the more complex behavior that emerges during development, as interneuronal projections increase in length and specificity.

\section{References}

Baskys A, Malenka RC (1991) Agonists at metabolic glutamate receptors presynaptically inhibit EPSCs in neonatal rat hippocampus. $J$ Physiol (Lond) 444:687-701.

Boppart SA, Wheeler BC, Wallace CS (1992) A flexible perforated microelectrode array for extended neural recordings. IEEE Trans BME 33:37-42.

Buzsáki G, Horváth Z, Urioste, R, Hetke J, Wise K (1994) Highfrequency network oscillation in the hippocampus. Scicnce 256: $1025-1027$

Charlety PJ, Jimbo Y, Maeda E, Kamioka H, Kawana A (1994) Developmental electrical activity changes in cultured cortical neural networks. Soc Neurosci Abstr 20:1299. 
Douglas WW (1968) Stimulus-secretion coupling: the concept and clues from chromaffin and other cells. Br J Pharmacol 34:451-474.

Droge MH, Gross GW, Hightower MH, Czinsy LE (1986) Multielectrode analysis of coordinated, multisite rhythmic bursting in cultured CNS monolayer networks. J Neurosci 6:1583-1592.

Engel AK, König P, Kreiter AK, Shillen TB, Singer W (1992) Temporal coding in the visual cortex: new vistas on integration in the nervous system. Trends Neurosci 15:218-226.

Forsythe ID, Clements JD (1990) Presynaptic glutamate receptors depress excitatory monosynaptic transmission between mouse hippo campal neurons. J Physiol (Lond) 429:1-16.

Gutnick MJ, Connors BW, Prince DA (1982) Mechanisms of neocortical epileptogenesis in vitro. J Neurophysiol 48:1321-1335.

Habets AMMC, Van Dongen AMJ, Van Huizen F, Corner MA (1987) Spontaneous neuronal firing patterns in fetal rat cortical networks during development in vitro: a quantitative analysis. Exp Brain Res 69:43-52.

Hamill OP, Marty A, Neher E, Sakmann B, Sigworth FJ (1981) Improved patch-clamp techniques for high-resolution current recording from cell and cell-free membrane patches. Pflugers Arch 391:85-100.

Huettner JE, Baughman RW (1986) Primary culture of identified neurons from the visual cortex of postnatal rats. J Neurosci 6:3044-3060.

Jimbo Y, Robinson HPC, Kawana A (1993) Simultaneous measurement of intracellular calcium and electrical activity from patterned neural networks in culture. IEEE Trans BME 40:804-810.

Kamioka H, Maeda E, Torimitsu K, Kawana A (1993) Developmental changes in activity of cultured cortical neural networks (abstract). Neurosci Res Suppl 18:S112.

Kuroda Y, Ichikawa M, Muramoto K, Kobayashi K, Matsuda K, Ogura A, Kudo Y (1992) Block of synapse formation between cerebral cortical neurons by a protein kinase inhibitor. Neurosci Lett 135:255258.

Kuroda Y, Muramoto K, Kawahara M, Kobayashi K, Sekino Y, Maeda E, Kawana A (1993) Spatial and temporal changes of patterns of synchronous oscillation in cultured cortical neuronal network by stimulation. Soc Neurosci Abstr 19:413.

Maeda E, Kawana A (1994) Generation and propagation of periodic spontaneous bursts in cultured cortical network. First Asian Symp Biophys 142.

Maeda E, Kuroda Y, Kawana A (1993) Transient changes of network activity in cultured cortical neurons induced by $\mathrm{Mg}^{2+}$ and APV (abstract). Neurosci Res Suppl 18:S233.

Maeda E, Robinson HPC, Kuroda Y, Kawana A (1993) Synaptic potentiation in cultured cortical neurons: long-lasting modification of spontaneous synchronized periodic firing by patterned electrical stimulation. Soc Neurosci Abstr 19:1323

Maeda E, Kuroda Y, Kawana A (1994) Long-term modification of network activity in cortical cultures elicited by synchronized bursts (abstract). Neurosci Res Suppl 19:S164.

Mayer ML, Westbrook GL, Guthrie PB (1984) Voltage-dependent block by $\mathrm{Mg}^{2+}$ of NMDA responses in spinal cord neurons. Nature 309:261-263.

McCormick DA, Prince DA (1987) Post-natal development of electrophysiological properties of rat cerebral cortical pyramidal neurons. $J$ Physiol (Lond) 393:743-762.

Meister M, Wong ROL, Baylor DA, Shatz CJ (1991) Synchronous bursts of action potentials in ganglion cells of the developing mammalian retina. Science 252:939-943.

Muramoto K, Kobayashi K, Nakanishi S, Matsuda Y, Kuroda Y (1988) Functional synapse formation between cultured neurons of rat cerebral cortex. Proc Jpn Acad Ser B Phys Biol Sci 64:319-322.

Murphy TH, Blatter LA, Wier WG, Baraban JM (1992) Spontaneous synchronous synaptic calcium transients in cultured cortical neurons. J Neurosci 12:4843-4845.

Nedergaard M (1994) Direct signaling from astrocytes to neurons in cultures of mammalian brain cells. Science 263:1768-1771.

Nicolls JG, Martin AR, Wallace BG (1992) From neuron to brain (3rd ed). Sunderland, MA: Sinauer.

Nowak L, Bregestovksi P, Asher P, Heret A, Prochiantz A (1984) Magnesium gates glutamate-activated channels in mouse central neurons. Nature 307:462-465.

Ogura A, Iijima T, Amano T, Kudo Y (1987) Optical monitoring of excitatory synaptic activity between cultured hippocampal neurons by a multi-site $\mathrm{Ca}^{2+}$ fluometry. Neurosci Lett 78:69-74.

Peinado A, Yuste R, Katz L (1993) Extensive dye coupling between rat neocortical neurons during the period of circuit formation. Neuron $10: 103-114$

Robinson HPC, Torimitsu K, Kuroda Y, Kawana A (1992) Characterization of spontaneous synchronized calcium transients in cultured networks of cerebral cortical neurons (abstract). Biophys J 61:A511.

Robinson HPC, Kawahara M, JimboY, Torimitsu K, Kuroda Y, Kawana A (1993) Periodic synchronized bursting and intracellular calcium transients elicited by low magnesium in cultured cortical neurons. J Neurophysiol 70:1606-1616.

Shatz CJ (1990) Impulse activity and the patterning of connections during CNS development. Neuron 5:745-756.

Silva LR, Amitai Y, Connors BW (1991) Intrinsic oscillations of neocortex generated by layer 5 pyramidal neurons. Science 251:432-435.

Steriade M, Nuñez A, Amzica $\mathbf{F}$ (1993) A novel slow $(<1 \mathrm{~Hz})$ oscillation of neocortical neurons in vivo: depolarizing and hyperpolarizing components. J Neurosci 13:3252-3265.

Stevens CF, Tsujimoto I' (1995) Estimates for the pool size of releasable quanta at a single central synapse and for the time required to refill the pool. Proc Natl Acad Sci USA 92:846-849.

Wheeler BC, Novak JL (1986) Current source density estimation using microelectrode array data from the hippocampal slice preparation. IEEE Trans BME 33:1204-1212.

Wong ROL, Meister M, Shatz CJ (1993) Transient period of correlated bursting activity during development of the mammalian retina. Neuron 11:923-938.

Yuste R, Peinado A, Katz LC (1992) Neuronal domains in developing neocortex. Science 257:665-669. 